\title{
Sound Localization in Toddlers with Normal Hearing and with Bilateral Cochlear Implants Revealed Through a Novel "Reaching for Sound" Task
}

DOI: $10.3766 /$ jaaa.18092

Erica E. Bennett* ${ }^{*}$

Ruth Y. Litovsky†

\begin{abstract}
Background: Spatial hearing abilities in children with bilateral cochlear implants (BiCls) are typically improved when two implants are used compared with a single implant. However, even with BiCls, spatial hearing is still worse compared to normal-hearing $(\mathrm{NH})$ age-matched children. Here, we focused on children who were younger than three years, hence in their toddler years. Prior research with this age focused on measuring discrimination of sounds from the right versus left.
\end{abstract}

Purpose: This study measured both discrimination and sound location identification in a nine-alternative forced-choice paradigm using the "reaching for sound" method, whereby children reached for sounding objects as a means of capturing their spatial hearing abilities.

Research Design: Discrimination was measured with sounds randomly presented to the left versus right, and loudspeakers at fixed angles ranging from $\pm 60^{\circ}$ to $\pm 15^{\circ}$. On a separate task, sound location identification was measured for locations ranging from $\pm 60^{\circ}$ in $15^{\circ}$ increments.

Study Sample: Thirteen children with BiCls (27-42 months old) and fifteen age-matched (NH).

Data Collection and Analysis: Discrimination and sound localization were completed for all subjects. For the left-right discrimination task, participants were required to reach a criterion of $4 / 5$ correct trials $(80 \%)$ at each angular separation prior to beginning the localization task. For sound localization, data was analyzed in two ways. First, percent correct scores were tallied for each participant. Second, for each participant, the root-mean-square-error was calculated to determine the average distance between the response and stimulus, indicative of localization accuracy.

Results: All $\mathrm{BiCl}$ users were able to discriminate left versus right at angles as small as $\pm 15^{\circ}$ when listening with two implants; however, performance was significantly worse when listening with a single implant. All $\mathrm{NH}$ toddlers also had $>80 \%$ correct at $\pm 15^{\circ}$. Sound localization results revealed root-mean-square errors averaging $11.15^{\circ}$ in $\mathrm{NH}$ toddlers. Children in the $\mathrm{BiCl}$ group were generally unable to identify source location on this complex task (average error $37.03^{\circ}$ ).

Conclusions: Although some toddlers with $\mathrm{BiCls}$ are able to localize sound in a manner consistent with $\mathrm{NH}$ toddlers, for the majority of toddlers with $\mathrm{BiCls,}$ sound localization abilities are still emerging.

Key Words: bilateral, children, cochlear implants, localization

Abbreviations: ANOVA = analyses of variance; $\mathrm{BiCls}=$ bilateral cochlear implants; $\mathrm{Cl}=$ cochlear implant; MAA = minimum audible angle; $\mathrm{NH}=$ normal hearing; RMS = root-mean-square; SRM = spatial release from masking; $\mathrm{SD}=$ standard deviation

\footnotetext{
*Henry Ford Health System, Detroit, Ml; †Waisman Center, University of Wisconsin-Madison, Madison, WI Center from the NIH-NICHD (Grant No. P30 HD03352). 


\section{INTRODUCTION}

$\mathrm{S}$ patial hearing abilities are vital for young children, particularly when learning to communicate in complex auditory environments. More specifically, sound localization abilities allow children to identify sound source location in environments like the classroom. To date, studies have typically been conducted with auditory stimuli placed along the horizontal plane (for review see Litovsky, 2011), where binaural cues provide information regarding sound source location (Blauert, 1987; Middlebrooks and Green, 1991). Locating sound sources is a skill that develops early in life (Clifton et al, 1981; Perris and Clifton, 1988; Grieco-Calub and Litovsky, 2012). The acuity with which infants and children perceive differences in spatial locations has been measured primarily using a discrimination task, in which the listener indicates if the sound is located toward the left or right of midline. Newborn infants with normal hearing $(\mathrm{NH})$ are reported to turn their head in the correct direction in the majority of trials, within hours after birth (Clifton et al, 1981). Within a few months after birth, the acuity with which infants can discriminate sounds to the left versus right has been assessed with the minimum audible angle (MAA), whereby the angular separation from midline is varied, to estimate the smallest change in sound source angle that listeners can discriminate. MAA thresholds have been measured using a two-alternative forced-choice task, with either overt pointing behaviors (Grieco-Calub et al, 2008; Grieco-Calub and Litovsky, 2012) or head-orienting behaviors (Ashmead, Clifton, and Perris, 1987; Muir et al, 1989; Morrongiello and Rocca, 1990; Litovsky, 1997). MAA thresholds in NH infants improve dramatically during the first year of life (Muir et al, 1989). MAA thresholds continue to improve, reaching $\sim 5^{\circ}$ by 18 months of age and $1-2^{\circ}$ by five years of age, the latter being within the range of thresholds measured in adults (Litovsky, 1997; for review see Litovsky et al, 2012).

A second way to measure spatial hearing abisilities is to test sound localization. To measure whether children can identify the location of a sound, rather than discriminate between two options, more complex tasks are needed. Developmental studies on sound localization have primarily used closed-set response options with loudspeakers positioned along an arc in the frontal hemifield. The increments separating loudspeakers vary across studies, being as small as 10 $15^{\circ}$ in some (e.g., Grieco-Calub and Litovsky, 2010; Zheng et al, 2015; Dorman et al, 2016) and as large as $45^{\circ}$ (Cullington et al, 2017). Behavioral measures include overt pointing or computer games requiring children to select options that indicate perceived location of a sound source. To assess sound localization abilities, two measures are fairly common: percent correct and root-mean-square (RMS) error, the latter being a statistical estimate of the average deviation of responses from the actual sound location. To date, the youngest age for which sound localization RMS errors have been reported is four to five years, and findings range from 6 to $29^{\circ}$ (Dorman et al, 1997; Van Deun, Van Wieringen, et al, 2009; Grieco-Calub and Litovsky, 2010; Zheng et al, 2015; Dorman et al, 2016). Lack of prior studies on localization of RMS errors in younger children are most likely due to the lack of appropriate tasks to elicit information from young listeners about where a sound source is perceived to be coming from.

Sound localization is of great interest as a measure of emerging spatial hearing abilities in patients who are deaf and receive bilateral cochlear implants (BiCIs). The interest is rooted in the fact that, having access to sound in both ears is thought to enable patients to localize better than if they only have access to sound in a single ear (for review see Litovsky, 2015; Litovsky and Gordon, 2016). Several studies in children with BiCIs have shown that the ability to discriminate sound location (left versus right) is indeed better when listening with two implants compared with only a single implant (Litovsky et al, 2006a; Grieco-Calub and Litovsky, 2010; Dorman et al, 2016). In addition, sound localization errors are smaller with two implants versus a single implant (e.g., Grieco-Calub and Litovsky, 2010; Cullington et al, 2017). These abilities have been shown to improve with bilateral hearing experience (e.g., Godar and Litovsky, 2010; Zheng et al, 2015). In addition, sound localization abilities appear to be better in children with BiCIs who received the implants in the two ears with brief delays compared with children who received the two implants several years apart (Chadha et al, 2011; Gordon et al, 2013a,b; for review see Litovsky and Gordon, 2016).

A common thread in studies with bilaterally implanted children is that regardless of the delay in implantation between the ears, they generally perform more poorly than $\mathrm{NH}$ peers (Van Deun, van Wieringen, et al, 2009; Grieco-Calub and Litovsky, 2010; Misurelli and Litovsky, 2012; 2015; Zheng et al, 2015; Dorman et al, 2016; Cullington et al, 2017; Reeder et al, 2017). A notable feature in previous studies is that children were aged four years or older, and in many cases, their hearing was not bilaterally activated until age three years or older. The focus of the present study was spatial hearing in younger children (toddlers), who were bilaterally activated by age 18 months to better understand if earlier activation of bilateral hearing promotes spatial hearing abilities. The observer-based psychophysical procedure developed by Olsho et al (1987) was used to determine if sounds presented from left versus right could be 
discriminated by young bilateral listeners in a manner consistent with $\mathrm{NH}$ age-matched peers (Grieco-Calub et al, 2008; Grieco-Calub and Litovsky, 2012). Testing was conducted when the toddlers wore either bilateral implants or a single implant. The range of MAA thresholds observed averaged $37.4^{\circ}$, compared with $\mathrm{NH}$ peers who averaged $14.5^{\circ}$; because sound levels were roved to minimize monaural level cues, MAAs were higher than in aforementioned $\mathrm{NH}$ studies. There was a trend for lower MAAs in toddlers with $>12$ months of bilateral experience than in toddlers with $<12$ months experience. When listening with a single implant, none of the toddlers were able to discriminate left versus right.

Another aspect of spatial hearing that has also been studied in children with BiCIs is the ability to segregate speech from noise, also known as spatial release from masking (SRM). Some studies have reported that children with BiCIs show smaller SRM compared with chronologically age-matched NH children (Mok et al, 2009; Murphy et al, 2011; Misurelli and Litovsky, 2012; Misurelli, 2014). In these studies, most children were sequentially implanted and experienced a duration of unilateral listening before becoming bilaterally implanted. By contrast, toddlers who were bilaterally activated by 18 months of age attained SRM levels similar to age-matched peers (Hess et al, 2018).

Taken together, studies to date suggest that there is a combination of factors that contribute to spatial hearing abilities in bilaterally implanted children, including the age of bilateral activation, interimplant delay, and age at testing. The present study was motivated by another factor, which is the task used to measure spatial hearing abilities. Behavioral measurements of localization are challenging and limited to tasks that are appropriate for the age and cognitive ability of the subjects. Two issues related to test paradigm may play a role in results on spatial hearing tasks. First, in a prior study, when tested on the left-right discrimination task with visual reinforcement, not all toddlers were able to discriminate sound locations, even when tested at large angular separations (Grieco-Calub and Litovsky, 2012). It may be that the visual reinforcement paradigm did not provide ample motivation for two to three year olds, which makes it difficult to discern between disengagement from the task and difficulty of the task. To address this issue, Litovsky et al (2013) designed a novel paradigm aimed at engaging two to three-yearold children through a "reaching for sound" task, whereby children were trained to reach for sounding objects hidden behind a curtain and were rewarded for identifying the correct location of the sound. In that study, six toddlers with BiCIs were tested on a twoalternative forced-choice task, and all showed left-right discrimination above chance with loudspeakers placed at $\pm 15^{\circ}$. The "reaching for sound" paradigm was also successfully implemented recently in a speech discrim- ination study (Peng et al, 2019). A second consideration related to test paradigm concerns whether children in their toddler years have developed a spatial map, and can identify sound locations, beyond being able to discriminate sound direction. In the present study, we thus adapted the "reaching for sound" left-right discrimination task (Litovsky et al, 2013) to a nine-alternative forced-choice location identification. This is the first study in which spatial hearing abilities have been measured in two to three-year-old children with BiCIs and $\mathrm{NH}$. We tested the hypothesis that sound localization has emerged by two to three years of age and should, thus, be observed in NH children. By contrast, children with BiCIs were not expected to identify source locations despite being able to discriminate left versus right.

\section{METHODS}

\section{Participants}

The BiCI group had 13 participants with an average age of $34.5 \pm 3.8$ months. All BiCI participants had at least one year of experience with the first cochlear implant (CI) and $18.6 \pm 6.5$ months of experience with the second CI. All participants were native English speakers, had no diagnosed developmental disabilities, and used oral communication as the primary mode of communication. Table 1 shows individual and average demographic information, including age of implantation, bilateral experience, gender, etc. Of these 13 participants, six had been tested on the discrimination task by Litovsky et al (2013); seven additional participants were recruited for the larger $\mathrm{N}$ size. In addition, 15 typically developing $\mathrm{NH}$ toddlers participated with an average age of $31.3 \pm 3.0$ months (see Table 2 for demographic information and tympanometric screening results). According to the information provided by parents on a screening questionnaire, none of the participants had a history of hearing loss, middle ear problems, or developmental delays. The discrimination data for the NH group were published in 2013 and are replotted here with permission. The localization data presented here for both $\mathrm{BiCI}$ and $\mathrm{NH}$ children groups have not been previously published.

\section{Experimental Setup}

In the present study, we adapted the "reaching for sound" paradigm used by Litovsky et al (2013) to a nine-alternative forced-choice localization task. This method was implemented following extensive pilot testing. The "reaching for sound" paradigm tests localization in a well-lit room and engages the children in a hide-and-seek game. This paradigm was modeled after the "reaching in the dark" studies in which 
Table 1. Demographic Data for Children with BiCls

\begin{tabular}{|c|c|c|c|c|c|c|c|}
\hline $\begin{array}{l}\text { BiCl Participants } \\
(n=13)\end{array}$ & Sex & $\begin{array}{c}\text { Age } \\
\text { (months) }\end{array}$ & $\begin{array}{c}\text { Age at } \\
1^{\text {st }} \text { Implant } \\
\text { (months) }\end{array}$ & $\begin{array}{c}\text { Age at } 2^{\text {nd }} \\
\text { Implant (months) }\end{array}$ & $\begin{array}{c}\text { Bilateral } \\
\text { Experience (months) }\end{array}$ & RMS Error & Percent Correct \\
\hline$\overline{\mathrm{CIEP}}$ & $\bar{F}$ & 34 & 13 & 29 & 5 & $52.39^{\circ}$ & $21.82 \%$ \\
\hline CIEQ & $F$ & 34 & 10 & 11 & 23 & $40.52^{\circ}$ & $37.04 \%$ \\
\hline CIER & M & 38 & 19 & 19 & 19 & $42.62^{\circ}$ & $11.11 \%$ \\
\hline CIEY & $M$ & 37 & 12 & 12 & 25 & $42.17^{\circ}$ & $38.10 \%$ \\
\hline CIEZ & $M$ & 41 & 12 & 32 & 9 & $43.13^{\circ}$ & $15.56 \%$ \\
\hline CIFB & $\mathrm{F}$ & 39 & 20 & 20 & 19 & $37.17^{\circ}$ & $19.44 \%$ \\
\hline $\mathrm{CIFI}$ & M & 32 & 7 & 7 & 25 & $41.53^{\circ}$ & $16.67 \%$ \\
\hline CIFJ & M & 30 & 14 & 14 & 16 & CNT & CNT \\
\hline CIFK & M & 30 & 14 & 14 & 16 & CNT & CNT \\
\hline CIFN & M & 34 & 13 & 13 & 21 & $32.02^{\circ}$ & $50.00 \%$ \\
\hline CIFQ & M & 28 & 7 & 13 & 15 & CNT & CNT \\
\hline CIFT & M & 36 & 8 & 8 & 28 & $10.98^{\circ}$ & $57.14 \%$ \\
\hline CIFU & $F$ & 36 & 12 & 15 & 21 & $27.84^{\circ}$ & $5.56 \%$ \\
\hline$A V G \pm S D$ & $\mathrm{~N} / \mathrm{A}$ & $34.5 \pm 3.8$ & $12.4 \pm 4.0$ & $15.9 \pm 7.4$ & $18.6 \pm 6.5$ & $37.0 \pm 11.3$ & $27.2 \pm 17.3$ \\
\hline
\end{tabular}

rudimentary spatial hearing was measured in infants (Perris and Clifton, 1988). Because pilot testing revealed that young children who are deaf were not testable in a dark room, we developed the "reaching for sound" in a lit room. This approach was designed to bear similarity to situations encountered during daily activities. Finally, the child-friendly nature of the setup has the potential to be implemented in a clinical setting for testing sound localization in children aged two to three years.

Testing was conducted in a standard Industrial Acoustics Corporation Acoustics sound booth $(2.7 \mathrm{~m} \times$ $3.6 \mathrm{~m}$ ). The testing apparatus consisted of a semicircular table (radius of $0.6 \mathrm{~m}$ ) with a curtain suspended above the table, as seen in Figure 1. The apparatus had a vertical $(30 \mathrm{~cm})$ wooden arc with nine holes, spaced $15^{\circ}$ apart from $-60^{\circ}$ to $60^{\circ}$ (left to right). Each hole had a diameter of $11 \mathrm{~cm}$, large enough for a child to reach their hand through. Loudspeakers (Cambridge SoundWorks Center/Surround IV, North Andover, MA) were positioned under each hole (at ear level when the child was seated) and were hidden by acoustically transparent curtains, hence the loudspeakers remained invisible to the child. The child sat on a chair facing the apparatus so that the array of holes in the curtain were visible and the center location was directly in front. During testing (described later), one of five different curtains with holes cut out at different angular separations was used to create loudspeaker configurations. A second curtain hid an experimenter who sat behind the apparatus. Two video cameras were used to track the child's responses. One video camera was positioned behind the child to track the child's behavior, whereas the other was positioned above the speaker at $0^{\circ}$ to track

Table 2. Demographic Data for Children with NH

\begin{tabular}{|c|c|c|c|c|}
\hline $\begin{array}{l}\text { NH Participants } \\
(n=15)\end{array}$ & Age & Tympanometric Screening & RMS Error & Percent Correct \\
\hline$\overline{\mathrm{COO}}$ & 31 & Pass & $33.17^{\circ}$ & $33.33 \%$ \\
\hline COR & 24 & Child would not participate & $14.43^{\circ}$ & $92.59 \%$ \\
\hline $\cos$ & 31 & Pass & $10.00^{\circ}$ & $66.67 \%$ \\
\hline COT & 31 & No tymp R; Peak Ytm $=0.2 \mathrm{~L}$ & $4.08^{\circ}$ & $92.59 \%$ \\
\hline $\mathrm{COQ}$ & 31 & No tymp L; Flat R & CNT & CNT \\
\hline COV & 33 & WNL R; Peak Ytm $=0.2 \mathrm{~L}$ & $0.00^{\circ}$ & $100 \%$ \\
\hline cow & 32 & Pass & $5.10^{\circ}$ & $88.46 \%$ \\
\hline cox & 34 & Low peaks Au & $35.59^{\circ}$ & $14.81 \%$ \\
\hline $\mathrm{COZ}$ & 25 & Pass & CNT & CNT \\
\hline CPF & 34 & Pass & $2.89^{\circ}$ & $93.60 \%$ \\
\hline $\mathrm{CPH}$ & 34 & No tymp L; WNL R & $8.66^{\circ}$ & $83.33 \%$ \\
\hline CPK & 33 & WNL L; PE tube R & $2.89^{\circ}$ & $96.30 \%$ \\
\hline CQC & 33 & Pass & $13.69^{\circ}$ & $83.33 \%$ \\
\hline CQD & 32 & Pass & $10.41^{\circ}$ & $74.07 \%$ \\
\hline CQE & 32 & Pass & $4.08^{\circ}$ & $93.59 \%$ \\
\hline$A V G \pm S D$ & $31.3 \pm 3.0$ & $\mathrm{~N} / \mathrm{A}$ & $11.15 \pm 11.2$ & $77.9 \pm 25.9$ \\
\hline
\end{tabular}




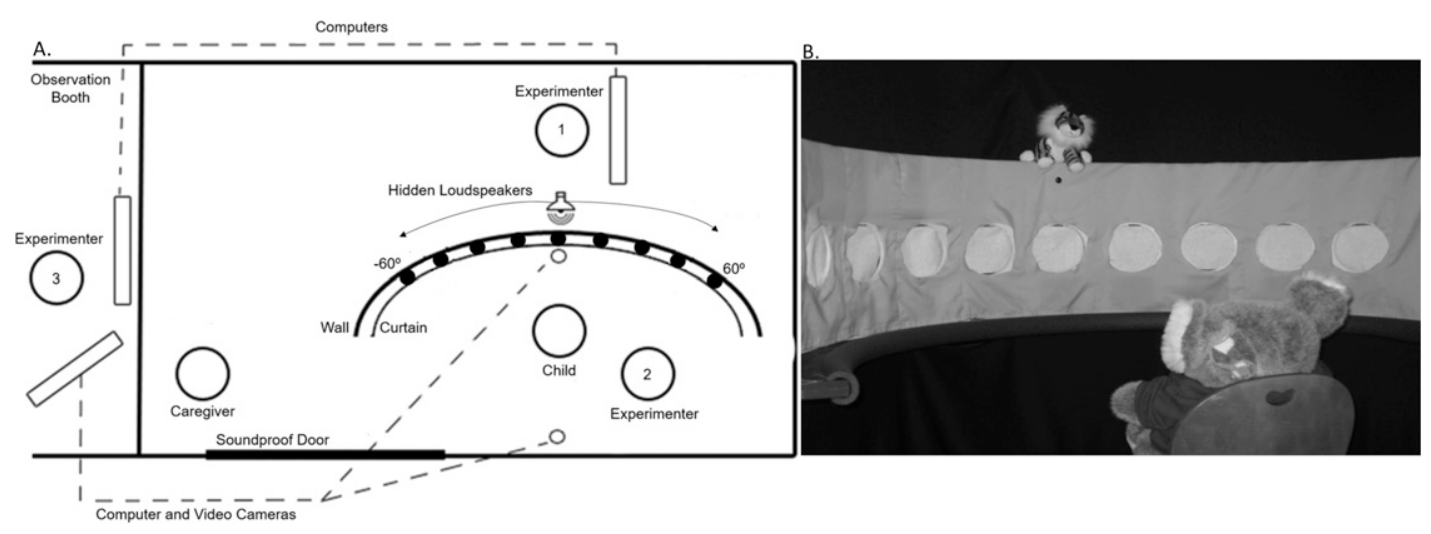

Figure 1. Diagram of the task apparatus depicting the setup for the sound localization study (panel A) and a photo of the actual sound localization setup (panel B).

eye gaze and head orientation. Audio stimuli were stored as .wav files and played to the loudspeakers through Tucker Davis System hardware (TDT). Customized software for running the program and data collection were written in the MATLAB programming language (The MathWorks, Natick, MA). This test setup is consistent with the experimental conditions in Litovsky et al (2013).

\section{Stimuli}

Acoustic stimuli consisted of a recorded female voice saying the carrier phrase "When I hide I say" followed by three bursts of white noise. Sounds were presented at $60 \mathrm{~dB}(\mathrm{~A}) \mathrm{SPL}$ and were proved to be varied $\pm 4 \mathrm{~dB}$ from the $60 \mathrm{~dB}$ to minimize monaural level cues, thus forcing participants to rely on binaural cues only. The level rove was selected to be consistent with prior studies in this laboratory (Litovsky et al, 2006; Litovsky et al, 2006; Grieco-Calub and Litovsky, 2010).

\section{Procedure}

\section{Familiarization}

Before beginning the left-right discrimination task, the toddler was familiarized using a puppet show game. Familiarization was designed so that upon hearing "When I hide I say," the toddler became accustomed to reaching for a hidden toy.

\section{Left-Right Discrimination Task}

Testing began with a discrimination test (Litovsky et al, 2013) in which the angles of separation were fixed, thus the participant only had two locations at the same angle on the left and the right as possible choices. To create this setup, a curtain revealing only the two locations being tested was placed on the apparatus (i.e., $\left.\pm 60^{\circ}\right)$. Left and right locations were pseudorandomized, meaning that the stimulus could not be presented from either side more than twice in a row. This was determined necessary after pilot testing, to prevent a toddler from perseverating to one side. Testing began with the widest angular separation of $120^{\circ}\left( \pm 60^{\circ}\right)$. The toddler was trained by experimenter 2 through directed reaches (hand-over-hand reaching) as well as visual pairs (toy being presented through the correct hole while the stimulus is playing). The length of training depended on the child's behavior and ability to adapt to the task. The goal was to train the toddler to understand that after a stimulus was presented, if they reached through the hole above the correct loudspeaker, there was a reward waiting for them (e.g., toy and puzzle piece). Once the toddler was trained to reach on his/her own, a criterion of $4 / 5$ correct trials ( $80 \%$ ) must obtained before testing at a smaller angular separation of $\pm 30^{\circ}$. If passed at $\pm 30^{\circ}$, then testing was also conducted at $\pm 15^{\circ}$. If failed at $\pm 30^{\circ}$, then the angle was increased to $\pm 45^{\circ}$. If passed at $\pm 45^{\circ}$, then $\pm 30^{\circ}$ was repeated; if that passed, then testing was conducted at $\pm 15^{\circ}$.

Three experimenters participated in administering each test session. Experimenter 1 was hidden behind the apparatus, and at the beginning of each trial, they positioned a small puppet toy above the center loudspeaker $\left(0^{\circ}\right)$ to attract the toddler's attention. This experimenter initiated stimulus presentation once the toddler's eye gaze was directed toward the center. The computer program determined the random order in which stimuli were randomly presented from the left or right loudspeaker, and thus indicated to the experimenter where the hidden toy (reinforcer) should be placed. After stimulus onset, the toddler's task was to indicate if the sound was from the left or right, by reaching into the correct hole in the curtain, and if they were correct, the hidden toy was awaiting their grasp. Experimenter 2 sat with the toddler in front of the arc to center the toddler and provide training for the task. Finally, the third experimenter sat in an observation booth to code correct or incorrect responses. The trial ended after a 
clear reach was initiated or if a reach was not initiated within 10 seconds of stimulus offset, typically due to inattention or fussiness. The rule of 10 seconds was determined following pilot testing, as many children with CIs needed additional time with the task. If the parent was also in the testing booth (which some toddlers preferred), they were instructed not to coach or direct their child. This procedure is consistent with our prior study using this task (Litovsky et al, 2013).

\section{Sound Localization}

In addition to the left-right discrimination task completed in this study and in Litovsky et al (2013), a localization task was also completed. Participants who passed the discrimination test at $15^{\circ}$ proceeded to localization testing, during which all nine holes were revealed. Testing was conducted in blocks of trials with the nine locations presented in random order. This approach for measuring sound localization abilities has been used previously in a multitude of studies on spatial hearing in children (e.g., Van Deun et al, 2009; GriecoCalub and Litovsky, 2010; Litovsky and Godar, 2010; Zheng et al, 2015). Most children completed 27 trials with 3 repetitions per location. Pilot testing showed that spatial hearing accuracy was somewhat poor and that reinforcing only for a reach toward the exact location was not a realistic expectation, especially for children with BiCIs. Thus, reinforcement was given when the child reached either through the correct hole or within $15^{\circ}$ of the correct hole. Experimenter 3 entered information into the computer indicating which hole the child selected.

Data were analyzed in three ways. First, percent correct scores were tallied for each participant, defined strictly as the proportion of trials on which a valid reach occurred, and the child reached into the correct hole. Second, consistent with prior studies (e.g., Van Deun et al, 2009; Grieco-Calub and Litovsky, 2010; Litovsky and Godar, 2010; Zheng et al, 2015), for each participant, taking all trials with valid responses, RMS error was calculated as follows:

$$
R M S=\sqrt{\frac{1}{K} \sum_{k=1}^{k} \frac{1}{N_{k}} \sum_{i=1}^{N_{k}}\left(r_{k, i}-s_{k}\right)^{2}}
$$

Here, $K$ is the total number of sound sources, $\mathrm{N}_{k}$ is the number of trials at $k$ th source, $r_{k, i}$ is the listener's response to the $i$ th trial on which the source is presented. For each trial, the difference between response and stimulus reflects the localization accuracy. However, for repeated trials at a given stimulus location, the RMS

Table 3. Discrimination Data for Children with NH, BiCl (Litovsky et al, 2013), and BiCl (Present Study)

\begin{tabular}{|c|c|c|c|}
\hline \multicolumn{4}{|c|}{ (A) Litovsky et al (2013), $\mathrm{NH}$} \\
\hline$\overline{\text { Participant }}$ & 60 & 30 & 15 \\
\hline $\mathrm{N}=13$ & $\checkmark$ & $\checkmark$ & $\checkmark$ \\
\hline $\mathrm{COZ}$ & $\checkmark$ & $\checkmark$ & $x$ \\
\hline $\mathrm{COQ}$ & $\checkmark$ & $x$ & DNT \\
\hline
\end{tabular}

(B) Litovsky et al (2013), $N=6$

\begin{tabular}{|c|c|c|c|c|c|c|}
\hline \multirow[b]{2}{*}{ Participant } & \multicolumn{3}{|c|}{ Bilateral } & \multicolumn{3}{|c|}{ Unilateral } \\
\hline & 60 & 30 & 15 & 60 & 30 & 15 \\
\hline$\overline{\mathrm{CIEP}}$ & $\checkmark$ & $\sqrt{ }$ & $\sqrt{ }$ & $\sqrt{ }$ & $\sqrt{ }$ & $\bar{x}$ \\
\hline CIER & $\checkmark$ & $\checkmark$ & $\checkmark$ & $\checkmark$ & $\checkmark$ & $x$ \\
\hline $\mathrm{ClBF}$ & $\checkmark$ & $\checkmark$ & $\checkmark$ & $\checkmark$ & $x$ & DNT \\
\hline CIEZ & $\checkmark$ & $\checkmark$ & $\checkmark$ & $\checkmark$ & $x$ & DNT \\
\hline CIEY & $\checkmark$ & $\checkmark$ & $\checkmark$ & $x$ & DNT & DNT \\
\hline CIEQ & $\checkmark$ & $\checkmark$ & $\checkmark$ & $x$ & DNT & DNT \\
\hline \multicolumn{7}{|c|}{ (C) Present Study, $N=7$} \\
\hline & \multicolumn{3}{|c|}{ Bilateral } & \multicolumn{3}{|c|}{ Unilateral } \\
\hline Participant & 60 & 30 & 15 & 60 & 30 & 15 \\
\hline$\overline{\mathrm{CIFT}}$ & $\checkmark$ & $\sqrt{ }$ & $\sqrt{2}$ & $\sqrt{ }$ & $\sqrt{2}$ & $\checkmark$ \\
\hline CIFU & $\checkmark$ & $\checkmark$ & $\checkmark$ & $x$ & DNT & DNT \\
\hline CIFI & $\checkmark$ & $\checkmark$ & $\checkmark$ & DNT & DNT & DNT \\
\hline CIFN & $\checkmark$ & $\checkmark$ & $\checkmark$ & DNT & DNT & DNT \\
\hline CIFQ & $\checkmark$ & $\checkmark$ & $\checkmark$ & DNT & DNT & DNT \\
\hline CIFJ & $\checkmark$ & $x$ & DNT & DNT & DNT & DNT \\
\hline CIFK & $x$ & DNT & DNT & DNT & DNT & DNT \\
\hline
\end{tabular}


error includes both constant and variability error, which are determined by localization accuracy and reliability.

Finally, children with BiCIs required significantly more trials to reach criterion at each of the angles tested. One concern with the additional trials required to reach criterion is that children with BiCIs reached the $80 \%$ correct by chance, essentially creating a "false pass" at $\pm 15^{\circ}$. Therefore, a Monte Carlo simulation with 10,000 runs, and a moving window of $4 / 5$ correct, extended from 5 to 80 trials, was run for the criterion data.

\section{RESULTS}

$\mathrm{T}$ able 3 shows discrimination data for the $\mathrm{NH}$ and BiCI groups. NH data are summarized from Litovsky et al (2013) in 3A; 13 of 15 participants passed the leftright discrimination criterion at all angles tested.
Participant COZ passed at $60^{\circ}$ and $30^{\circ}$ but not at $15^{\circ}$. Participant COQ passed at $\pm 60^{\circ}$ but not at $\pm 30^{\circ}$. Results for BiCI users are shown in $3 \mathrm{~B}$ and $3 \mathrm{C}$. Data from the six children in the Litovsky et al (2013) study are shown in $3 \mathrm{~B}$ and seven additional children from the present study are shown in the bottom table (3C). Data are shown for bilateral and unilateral conditions for all participants with BiCIs. Outcomes differed among the participants, such that criterion was reached at different angles. In the bilateral listening mode, 10/13 participants reached the $80 \%$ criterion for all angles tested $\left(60^{\circ}, 30^{\circ}\right.$, and $15^{\circ}$ ), with no need to test at $45^{\circ}$ (as per the rules for selecting angles described earlier). Participant CIFJ met criterion at $60^{\circ}$ but not at $30^{\circ}$ and was not tested at $45^{\circ}$ because of lack of time. Participant CIFK did not meet criterion at $60^{\circ}$ and was not tested at smaller angles or in the unilateral condition. In the
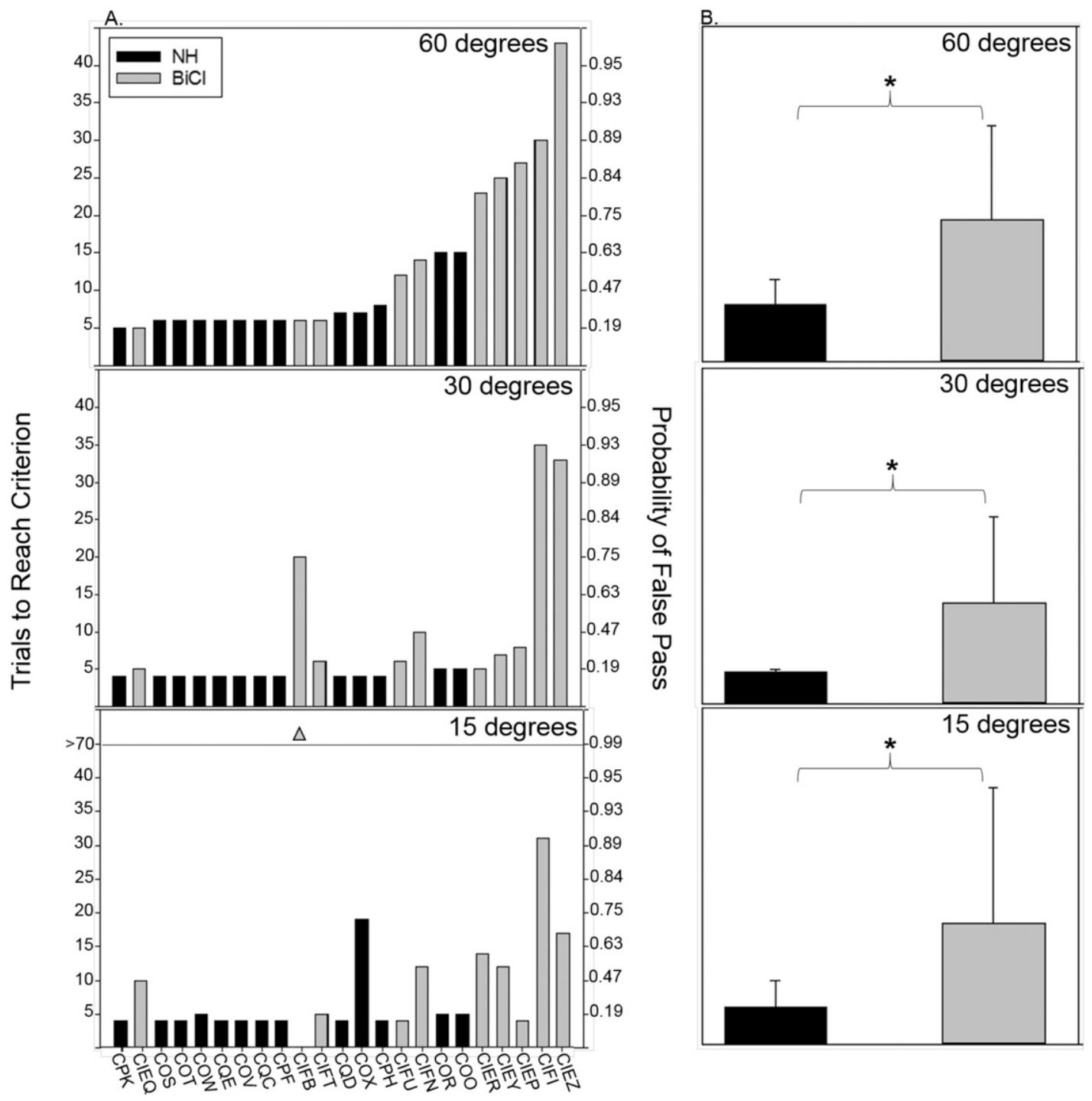

Figure 2. Number of trials to reach criterion are shown for individual children (panel A) and group data (average \pm SD; panel B). In panel $\mathrm{A}$, data from children with $\mathrm{NH}$ and with BiCIs are rank-ordered by number of trials. Children with $\mathrm{NH}$ and BiCIs are shown in dark and gray bars, respectively. 
unilateral listening mode, only one participant (CIFT) reached $80 \%$ criterion at all angles tested. Two participants (CIEP and CIER) reached $80 \%$ criterion at $60^{\circ}$ and $30^{\circ}$ but not at $15^{\circ}$, and two participants (CIEZ and CIBF) reached criterion at $60^{\circ}$ but not at smaller angles. Two participants (CIFI and CIFN) were not tested with a unilateral implant because of time constraints and/or loss of attention.

We were interested in whether the groups of children with BiCIs and with $\mathrm{NH}$ required a different length of time to reach criterion. Therefore, the number of trials to reach the criterion for the localization task $(>80 \%$ correct at $\pm 15^{\circ}$ ) was calculated. For both groups, the total number of trials to reach criterion at all angles tested $\left(60^{\circ}, 30^{\circ}\right.$, and $\left.15^{\circ}\right)$ was calculated. For the BiCI group, this value was calculated only for the participants who reached the criterion for all angle separations in the bilateral condition. Individual participants are shown in Figure 2A, rank ordered by the number of trials to reach criterion, from smallest to largest. The two groups are delineated with shading ( $\mathrm{NH}$ in black shade and BiCI in gray shade). Group averages ( \pm standard deviation [SD]) are shown in Figure $2 \mathrm{~B}$ and were as follows for $60^{\circ}, 30^{\circ}$, and $15^{\circ}: 7.6( \pm 3.4), 4.1( \pm 0.38)$, and 5.4 $( \pm 4.1)$ for $\mathrm{NH}$; $19.1( \pm 12.6), 13.5( \pm 11.7)$, and 18 $( \pm 20.3)$ for BiCI. Group comparisons with one-way, between-subjects analyses of variance (ANOVAs) for unequal $\mathrm{N}$ showed that the $\mathrm{BiCI}$ group required significantly more trials to reach criterion than the $\mathrm{NH}$ group at $60^{\circ}\left[F_{(1,21)}=10.062, p=0.005\right], 30^{\circ}$ $\left[F_{(1,21)}=8.442, p=0.008\right]$, and $15^{\circ}\left[F_{(1,21)}=4.85\right.$, $p=0.039]$. These results suggest that even though toddlers in the BiCI group (in this data set) were able to discriminate left versus right at angles as small as $\pm 15^{\circ}$, they required significantly more trials, or time, to complete this task. The additional trials needed to meet criteria may be related to task difficulty, as chil- dren with BiCIs may need additional experience with the task than their NH peers.

One issue for consideration is whether future studies are needed that limit the number of trials allowed before reaching criterion. The present study was designed primarily to determine whether the reaching method provides an ideal approach for determining spatial hearing abilities in toddlers. The method was designed to obtain percent correct of a running window of five trials. If each set of five trials is treated independently, then $4 / 5(80 \%)$ would be a good criterion. Pilot testing with several $\mathrm{NH}$ toddlers revealed the $4 / 5$ criterion to be appropriate. However, after initiation of the study with both groups, it was clear that some of the toddlers, in particular those with BiCIs, could only reach criterion if provided with additional trials. Because this method had never been applied to this population and because this population is difficult to recruit for testing, the decision was made to use a running window of $4 / 5$ trials. One must also consider the fact that the task (selecting a sound from the left versus right) is novel for these children and may require a certain amount of learning. In attempting to address this potential challenge in data interpretation, we conducted Monte Carlo simulations to estimate the probability of pass/fail given a running window of $4 / 5$, with an increase in number of attempts. Figure 2 showed the results of the Monte Carlo simulations. The $y$-axis label on the right side of Figure 2 shows values corresponding to the probability of false pass as a function of the number of trials needed to pass the criterion of $4 / 5$ correct. The false pass rate, e.g., reaches 0.25 with six trials, 0.46 with 10 trials, and 0.75 with 20 trials. Vertical comparison of individual subjects from $60^{\circ}$ to $30^{\circ}$ to $15^{\circ}$ provides insight into whether individual toddlers seemed to get more efficient at the task. For example, participants CIEP, CIEZ, CIER, and CIFT required higher levels to reach the criterion at $60^{\circ}$ than at either $30^{\circ}$

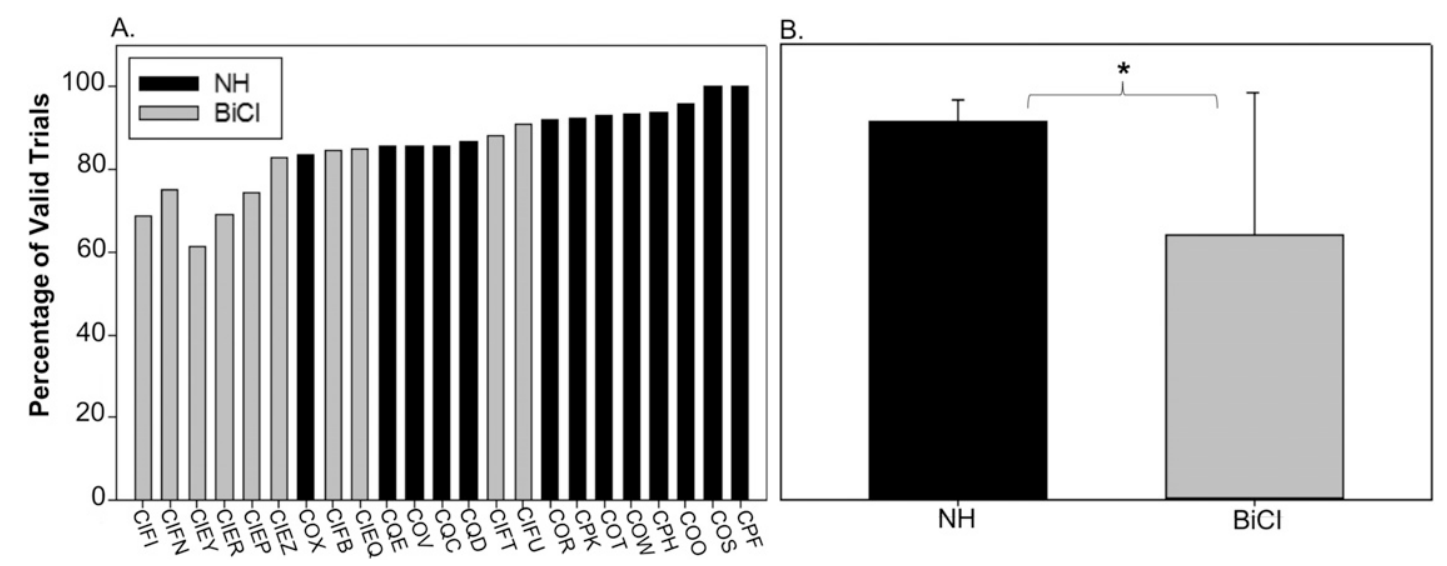

Figure 3. Percentages of valid trials are shown for individual children (panel A) and by group (average \pm SD; panel B). In panel A, data from children with $\mathrm{NH}$ and with BiCIs are rank-ordered by percent of valid trials. Children with NH and BiCIs are shown in dark and gray bars, respectively. 
or $15^{\circ}$, suggesting that as they gained more experience with the task, they were less likely to pass by chance and more efficient at reaching the $80 \%$ criterion. This analysis suggests that learning and experience may play an important role in outcomes with the reaching task. Future studies should also consider using a more stringent criterion than $4 / 5$ correct.

In this experiment, a trial was considered to be invalid if the child did not reach for the sound within 10 seconds following stimulus offset. In most cases, the child's failure to reach for the sound could be attributed to inattention or fussiness. Figure 3 shows the percent of trials that were considered valid for the two groups. Once again, for both groups, data are included only from toddlers who reached criterion for all locations. Individual data are shown in Figure 3A, rank ordered from lowest to highest in terms of percent of valid trials $(\mathrm{NH}=$ black, BiCI = gray). Group averages are shown in Figure 3B. Group averages were $91.33 \pm 0.05$ and $63.76 \pm$ 34.4 for NH and BiCI, respectively. A one-way betweensubjects ANOVA showed significantly fewer valid trials in the BiCI compared with the $\mathrm{NH}$ group $\left[F_{(1,21)}=\right.$ $8.125, p=0.01]$. This finding is related to the aforementioned result showing that $\mathrm{BiCI}$ users required significantly more trials to reach criterion.

Participants who met criterion at $\pm 15^{\circ}$ proceeded to the sound localization task. Localization accuracy for the $13 \mathrm{NH}$ participants is shown in Figure 4, with panels arranged lowest to highest RMS error. Individual participants' percent correct and RMS error values can be found in Table 2 . The majority of $\mathrm{NH}$ participants were able to localize sound with less than $15^{\circ}$ RMS error. Percent correct ranged from 14\% to $100 \%$ (77.9\% \pm $0.26 \%)$ and RMS errors ranged from 0 to $35^{\circ}\left(11.1^{\circ} \pm\right.$ $11.2^{\circ}$ ). Two participants (COO and COX) were not able to localize sounds, evidenced by generally reaching toward the center location, regardless of where the target was coming from.

Figure 5 shows the localization accuracy for the 10 participants with BiCIs who reached criterion on the left-right discrimination task, arranged from lowest to highest RMS error. For some participants, localization was in the range of performance seen in some of the NH participants (i.e., CIFT and CIFN). However, all others did not show evidence for spatial hearing as measured with this task. Percent correct ranged from $5.5 \%$ to $50 \%(27.2 \% \pm 0.17 \%)$ and RMS errors ranged from 10.9 to $52^{\circ}\left(37.1^{\circ} \pm 11.3^{\circ}\right)$; individual data are shown in Table 1. Figure 6 shows all the data combined for the two groups (BiCIs on the left, $\mathrm{NH}$ on the right). This figure shows that as a group, $\mathrm{NH}$ participants were more accurate in their responses to the target stimulus than BiCI participants. Figure 7 compares the two groups for percent correct (panel A) and RMS errors (panel B). One-way between-subjects ANOVAs revealed significantly lower percent correct in $\mathrm{BiCI}$ than $\mathrm{NH}\left[F_{(1,21)}=28.40, p<0.001\right]$ and significantly higher RMS errors in BiCI than $\mathrm{NH}\left[F_{(1,21)}=30.86\right.$,

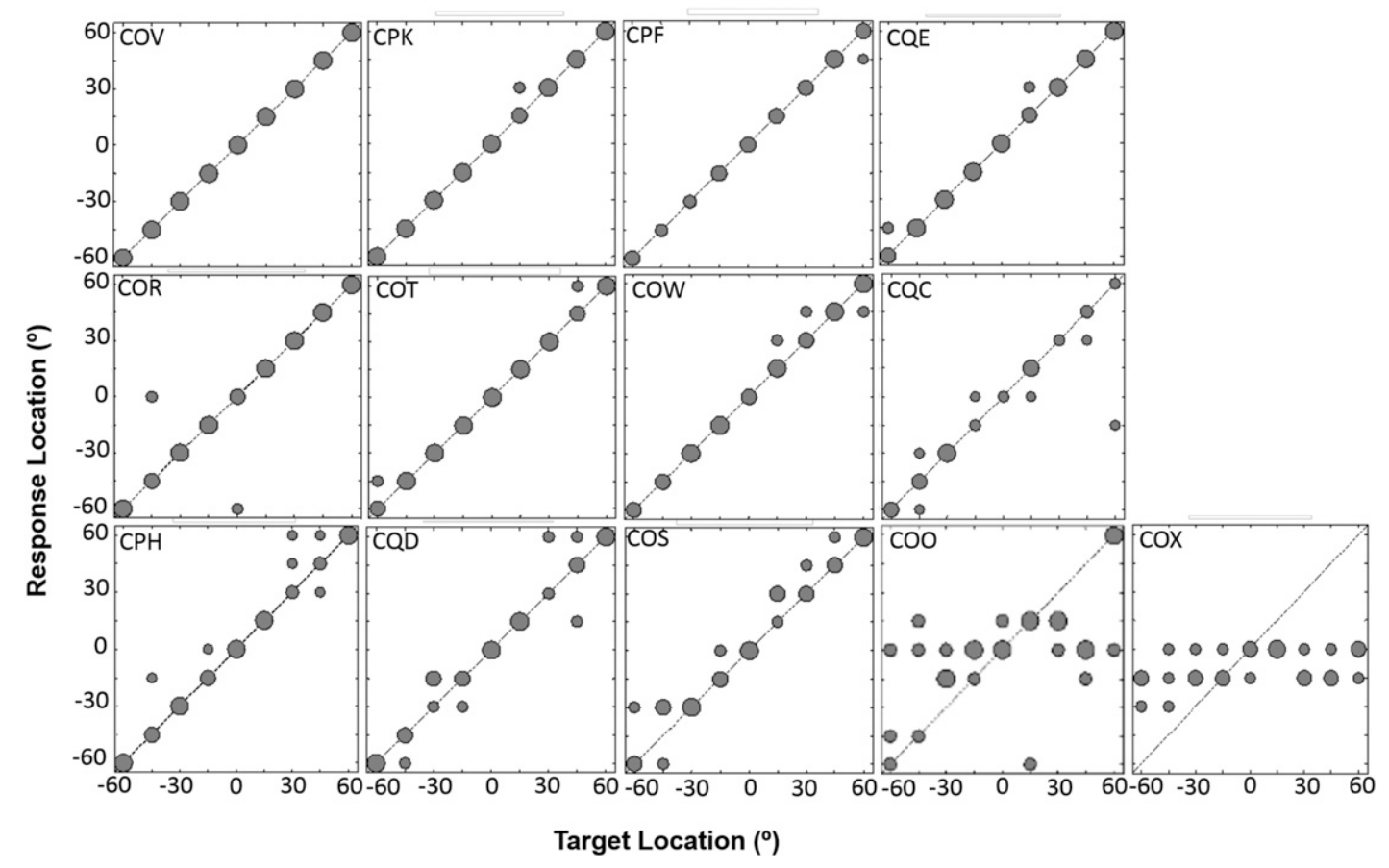

Figure 4. Individual performance on the sound localization task in the NH group. The size of the dots represents the number of responses for a given target location (i.e., the larger dots reflect a greater number of responses). The diagonal line is indicative of perfect performance. 


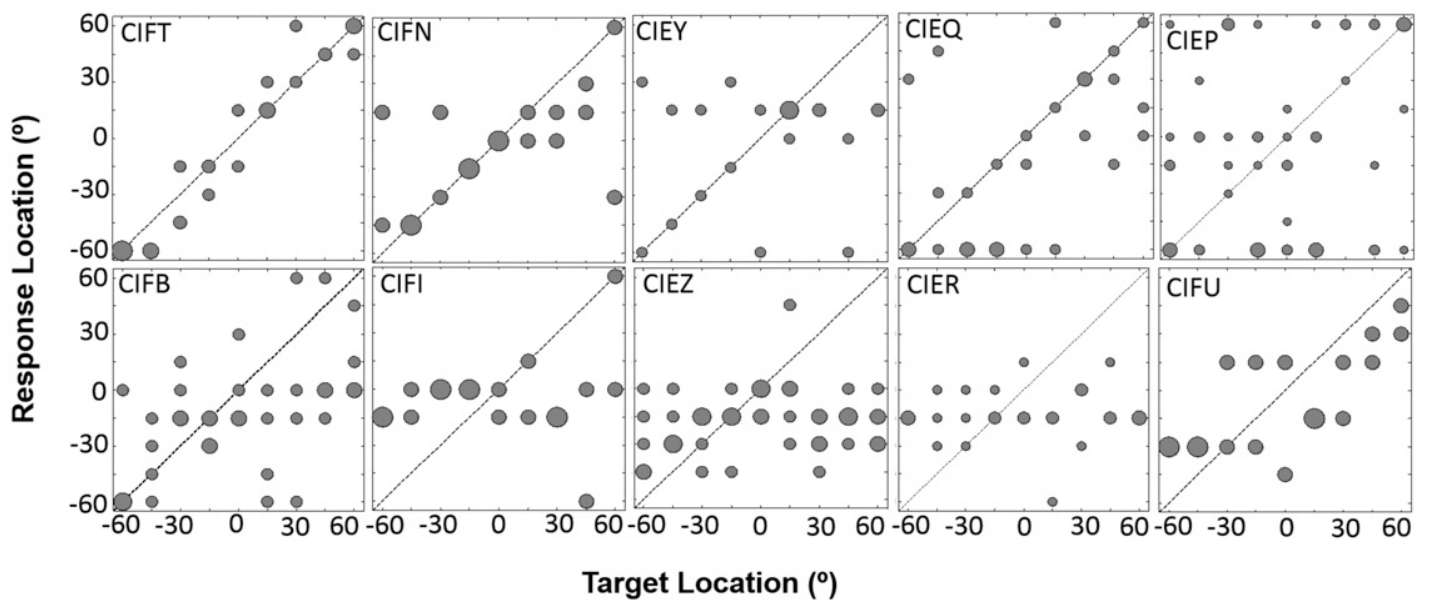

Figure 5. Individual performance on the sound localization task in the BiCI group. The size of the dots represents the number of responses for a given target location (i.e., the larger dots reflect a greater number of responses). The diagonal line is indicative of perfect performance.

$p<0.001]$. These significant differences suggest that although all participants could discriminate left-right at $15^{\circ}$, the ability did not transfer over to the sound location identification task.

\section{DISCUSSION}

$\mathrm{T}$ The present study investigated emerging spatial hearing abilities in two to three-year-old children (toddlers), in two groups defined as $\mathrm{NH}$ or with BiCIs. This is the first study to measure sound localization abilities in this age group. Past research has shown that $\mathrm{NH}$ infants aged five to six months can reach for sounding objects in the dark; when tested with target areas separated by $15^{\circ}$ increments, they were found to reach with $75 \%$ accuracy toward the correct target area (Perris and Clifton, 1988). Here, we also tested location identification, and we quantified percent correct as well as
RMS errors. Given the infant behavior documented in the literature, it is not surprising that percent correct in NH toddlers was $77.9 \%( \pm 0.26 \%)$ and RMS errors averaged $11.1^{\circ}\left( \pm 11.2^{\circ}\right)$.

The level of performance in the $\mathrm{NH}$ group in this study is within the range seen in older $\mathrm{NH}$ children (4+ years) tested using sound location identification tasks involving pointing to an icon on a computer screen matching the perceived location of the auditory object (e.g., Grieco-Calub and Litovsky, 2010; Zheng et al, 2015). The present finding suggests that sound localization abilities in some $\mathrm{NH}$ toddlers are well developed even when forced to rely on binaural cues, as monaural level cues were minimized with an overall level rove. However, variability in performance was observed with some NH toddlers (i.e., participants $\mathrm{COO}$ and $\mathrm{COX}$ ) performing in the lower range (percent correct lower than 50 and RMS above $30^{\circ}$ ). The range of performance seen
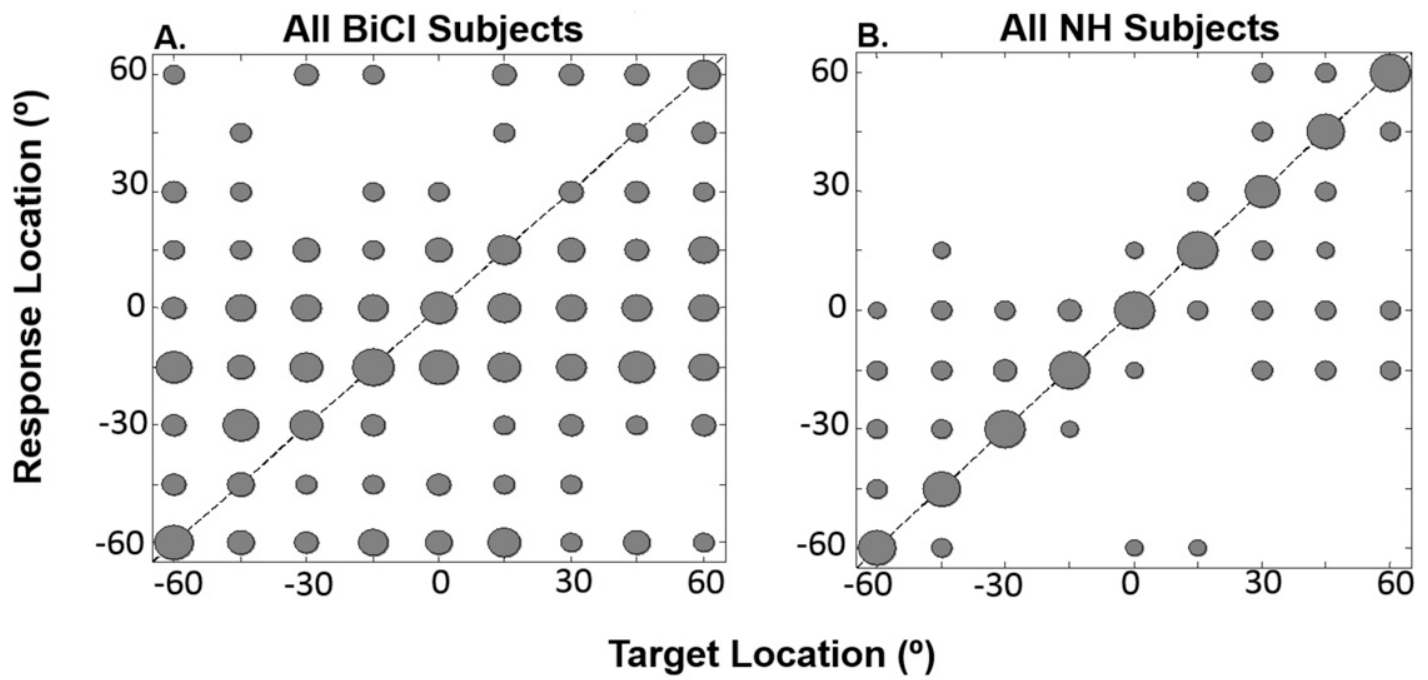

Figure 6. Group performance on the sound localization task for children with BiCIs (panel A) and children with NH (panel B). 

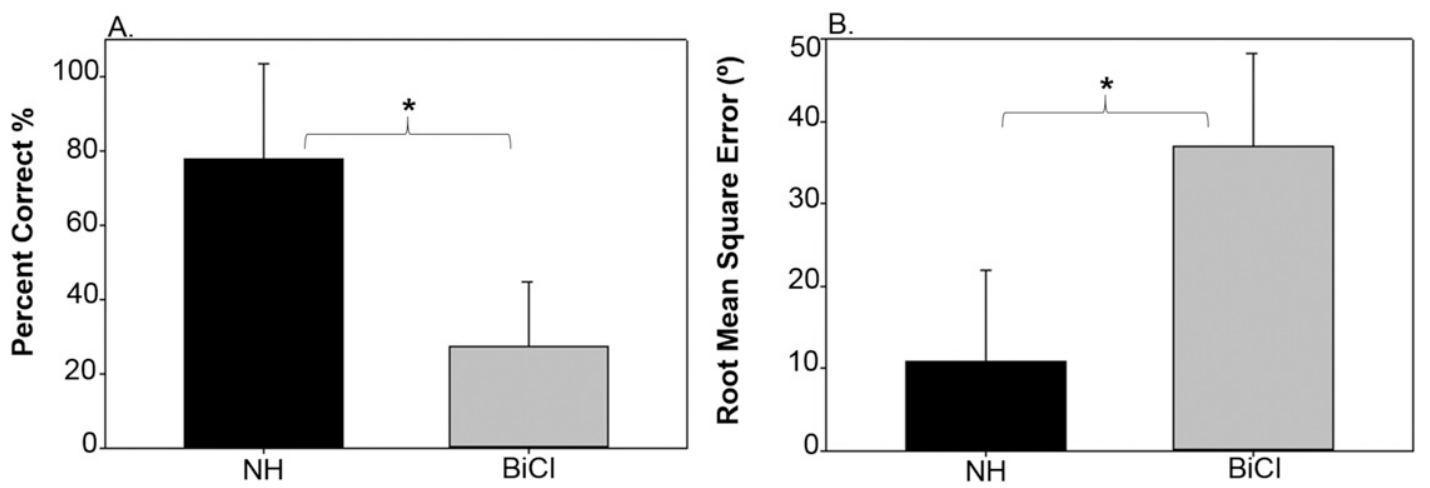

Figure 7. Group data for overall percent correct (panel A) and root mean square (RMS) error (panel B). Children with NH and BiCIs are shown in dark and grey bars, respectively. Significant differences are indicated with an asterisk $\left.{ }^{*}\right)$.

in the $\mathrm{NH}$ group suggests that spatial hearing abilities are still developing in some young children. Research has shown that the acoustic binaural cues required to perform on this task are developed by this age (Clifton et al, 1988), but this type of variability in spatial hearing has been observed previously (Litovsky, 1997; Grieoco-Calub and Litovsky, 2010). It may be that nonauditory factors such as memory, attention, and executive function play a role in the ability of young listeners to perform on the task. Nonetheless, having established the efficacy of the "reaching for sound" approach in $\mathrm{NH}$ toddlers (Litovsky et al, 2013), we sought to measure spatial hearing abilities in young, bilaterally implanted toddlers.

Previous research on spatial hearing abilities in bilaterally implanted toddlers (average age 2.5 years) used only a left-right discrimination task, whereby participants were reinforced for looking toward the correct hemifield (Grieco-Calub and Litovsky, 2012). In that prior study, although some toddlers with BiCIs performed similarly to $\mathrm{NH}$ peers, there was large variability in performance, with some bilaterally implanted toddlers showing very poor, or no ability, to discriminate left from right, even with large angular separations. Part of this variability may have been due to the task; "looking while listening" may be an excellent task for infants, but for toddlers who are active and mobile, this task may not have held this populations' attention, thus, it was not able to elicit reliable behaviors. Therefore, the "reaching for sound" method implemented. The present study is a follow-up to the original observation by Litovsky et al (2013) who measured left-right discrimination in six toddlers with BiCIs, all of whom reached $80 \%$ for nearly all angles tested in the bilateral listening mode. Here, we extended the reaching method to investigate discrimination in a larger group of participants and to test sound location identification, something that has not been performed before in this population.

Results from the discrimination task showed that in the bilateral listening mode, most toddlers with BiCIs reached criterion of $80 \%$ at the smallest angles tested of $\pm 15^{\circ}$. By contrast, in the unilateral listening mode, all toddlers with BiCIs (with the exception of participant CIFT) were either unable to perform the task at all or could only discriminate left versus right at largest angular separations. This finding is consistent with previous research in toddlers and in older children who are bilaterally implanted (Litovsky et al, 2006; Van Deun et al, 2009; Grieco-Calub and Litovsky, 2012). However, even in the bilateral condition, participants required significantly more practice (larger number of trials) to reach the criterion compared with the NH participants. In addition, participants had a larger number of invalid trials, suggesting a lack of attention, which may suggest difficulty with the task. The larger number of trials deemed to be invalid or trials on which the child did not reach for the sound, may be due to slower processing time and/or uncertainty about the stimulus. Slower response times and less accurate responses have also been reported for toddlers with BiCIs when word identification has been measured using an eye gaze paradigm (Grieco-Calub et al, 2009). In addition, research on reaction time and binaural fusion has shown that children with BiCIs demonstrate longer reaction time to stimuli that are not perceived as binaurally fused (Steel et al, 2015).

Because of the larger number of trials required to reach the criterion, we must consider a potential limitation in the design of the study, in that the large number of trials led to a false pass. During the discrimination task, we had a running window of $4 / 5$ correct to reach criterion. However, by extending the number of trials allowed for reaching the criterion, the probability of reaching criterion by chance increased. This more relaxed criterion likely contributed to the seemingly good performance of toddlers with BiCIs. However, if they were likely to reach criterion entirely by chance, they would have done so in the unilateral condition as well as in the bilateral condition, thus increasing the confidence that children with BiCIs are 
accurately able to discriminate left versus right at angles as small as $\pm 15^{\circ}$.

One factor contributing to limited performance in $\mathrm{BiCI}$ users is the fact that they had less auditory experience than their NH peers. In this study, BiCI users received the first implant by approximately 12 months of age, and the second implant averaged 15.9 months of age (see Table 1), which may have contributed to their need for more experience on the task. A potential limitation of this study is that the $\mathrm{NH}$ and $\mathrm{BiCI}$ group were chronically age-matched rather than matched by their hearing age. This was due to the limited range of ages appropriate for the task used in this study. If the authors were to age-match the groups based on hearing age, the age range tested would require that subjects be either younger than two or older than three years of age. The "reaching for sound" method has been shown to be appropriate for toddlers between the ages of two to three years both in the previous literature (Litovsky et al, 2013) and in pilot testing. Children younger than two years are not able to complete the reaching task as it is too difficult. In addition, children older than three years become bored with the task quickly as it is too easy.

This study was the first to test sound localization abilities in this population. Toddlers with BiCIs performed poorer than the NH toddlers on this task. The poor localization ability of toddlers with BiCIs is consistent with findings in older children with BiCIs; although they may have small MAA thresholds, the ability to discriminate between sounds from left or right is not predicative of the ability to localize sound (Grieco-Calub and Litovsky, 2010). When making comparisons with children with $\mathrm{NH}$, even the best performers (CIFT and CIFN) still had lower percent correct values and higher RMS errors than many of the NH children. Three participants (CIFN, CIFT, and CIFU) had RMS errors that fell within the range of $\mathrm{NH}$ toddlers. Participants CIFT and CIFN were both simultaneously implanted, participant CIFU had an interimplant delay of three months, and all three participants had bilateral experience of more than 20 months. Research using electrophysiologic measures suggests that early bilateral activation promotes neural responses in the auditory cortex that are similar to those seen in $\mathrm{NH}$ children, whereas longer interimplant delays or bilateral activation at later ages promote abnormal cortical activation patterns (Gordon et al, 2013b; 2015).

Two participants that showed difficulty with the task (percent correct was less than $30 \%$ and RMS error was higher than $40^{\circ}$ ) were participants CIEP and CIEZ. Participant CIEP had an interimplant delay of 16 months and participant CIEZ had an interimplant delay of 20 months. Children with a shorter interimplant delay (roughly 1.5 years or less) were more likely to demonstrate better sensitivity binaural cues than children with $>1.5$ years between activation of the two implants (Gordon et al, 2014). It may be that more experience with BiCIs is required for sound localization abilities to emerge. In fact, a recent study demonstrated this in older children, with results showing that children who had early auditory deprivation and shorter amounts of auditory experience with BiCIs were more likely to categorize sound as right versus left, whereas older children with longer auditory experience were able to map sound source locations (Zheng et al, 2015).

Based on those findings, we might have predicted that spatial hearing abilities would have matured in the young-implanted, early-activated toddlers and that perhaps they would function similarly to their $\mathrm{NH}$ peers. However, although early activation might promote the best opportunity for emergence of binaural processing as measured with electrophysiological responses, it does not guarantee that functional abilities are similar to those of $\mathrm{NH}$ participants. Although some toddlers with BiCIs were able to localize sounds, the majority showed difficulty with the task. The gap in performance between the $\mathrm{NH}$ and BiCI participants tested here could be due to the differences in access to binaural cues between the two groups. NH children receive acoustic cues that carry binaural information known to be important for sound localization, namely, interaural differences in level and time. The brain continually must recalibrate spatial mapping during development, as the head size grows (Clifton et al, 1988); however, $\mathrm{NH}$ infants are sensitive to those cues during the first year of life (Ashmead et al, 1991). By contrast, young listeners who are fitted with bilateral CIs do not receive binaural cues with fidelity, due to a number of limitations in the devices, including lack of synchronization between the two CI processors. Thus, BiCI users receive degraded binaural cues, which compromise sound localization abilities (Van Hoesel, 2004; Kan and Litovsky, 2015; Baumgärtel et al, 2017). Degraded binaural information may create poorly defined spatial maps in this population (Litovsky, 2011; Litovsky et al, 2012; Zheng et al, 2015) and make it difficult for young children with BiCIs to develop well-defined maps of auditory space. In addition, young BiCI users might require additional listening experience to acquire abilities more closely aligned with their $\mathrm{NH}$ peers, an issue that remains to be better understood.

In summary, toddlers with $\mathrm{NH}$ demonstrated discrimination and localization abilities similar to older children (e.g., Grieco-Calub and Litovsky, 2010; Zheng et al, 2015). By contrast, the group of toddlers with BiCIs, even when tested while wearing both implants, performed worse than their NH peers. Although they were able to discriminate source locations from left versus right, as has already been shown previously with two different methods (Grieco-Calub and Litovsky, 2012; Litovsky et al, 2013), identifying where sounds 
are located had not yet developed in these young listeners. These results suggest that spatial hearing is a developing skill in this population.

\section{CONCLUSIONS}

$\mathrm{S}$ patial hearing using two tasks, left-right discrimination and a sound location identification, was studied in two to three-year olds with $\mathrm{NH}$ and with BiCIs. This was the first study to demonstrate sound localization skills in two to theee-year-old $\mathrm{NH}$ toddlers that are within the range observed at five years and older. Toddlers with BiCIs performed significantly better on left-right discrimination tasks with both implants compared with a unilateral implant. Toddlers with BiCIs took significantly longer to achieve the same performance on the left-right discrimination task as their $\mathrm{NH}$ peers, suggesting that further exposure to the task may be required. In addition, some toddlers with BiCIs are able to localize sound in a manner consistent with $\mathrm{NH}$ toddlers; however, for most toddlers with BiCIs, sound localization abilities are a skill that is still emerging. Further research is necessary with a larger sample to continue to understand the development of spatial hearing in toddlers with BiCIs.

Acknowledgments. The authors express their gratitude to the children and parents who participated in this study, to Shelly Godar, Christi Hess, Sara Misurelli, and Samantha Harris for assistance in data collection and recruitment of participants, and to Emily Burg and Sara Misurelli for providing input on an earlier draft of the manuscript. We are also very grateful to Z. Ellen Peng for comments on an earlier version of the paper and for help with the Monte Carlo Simulations.

\section{REFERENCES}

Ashmead D, Clifton RK, Perris E. (1987) Precision of auditory localization in human infants. Dev Psychol 23(5):641-647.

Ashmead D, Davis D, Whalen T, Odom R. (1991) Sound localization and sensitivity to interaural time differences in human infants. Child Dev 62:1211-1226.

Baumgärtel R, Hu H, Kollmeier B, Dietz M. (2017) Extent of lateralization at large interaural time differences in simulated electric hearing and bilateral cochlear implant users. J Acoust Soc Am 141(4):2338-2352.

Blauert J. (1987) Spatial hearing: the psychophysics of human sound localization by Jens Blauert. J Acoust Soc Am 77(1):334-335.

Chadha N, Papsin B, Jiwani S, Gordon K. (2011) Speech detection in noise and spatial unmasking in children with simultaneous versus sequential bilateral cochlear implants. Otol Neurotol 32(7): 1057-1064.

Clifton R, Morrongiello B, Kulig J, Dowd J. (1981) Newborns' orientation toward sound: possible implications for cortical development. Child Dev 52(3):833-838.
Clifton R, Gwiazda J, Bauer J, Clarkson MG, Held RM. (1988) Growth in head size during infancy: implications for sound localization. Dev Psychol 24:477-483.

Cullington HE, Bele D, Brinton JC, Cooper S, Daft M, Harding J, Hatton N, Humphries J, Lutman ME, Maddocks J, Maggs J, Millward K, O’Donoghue G, Patel S, Rajput K, Salmon V, Sear T, Speers A, Wheeler A, Wilson K. (2017) United Kingdom national paediatric bilateral project: results of professional rating scales and parent questionnaires. Cochlear Implants Int 18(1): 23-35.

Dorman M, Loizou P, Rainey D. (1997) Speech intelligibility as a function of the number of channels of stimulation for signal processors using sine-wave and noise-band outputs. J Acoust Soc Am 102(4):2403-2411.

Dorman MF, Loiselle LH, Cook SJ, Yost WA, Gifford RH. (2016) Sound source localization by normal-hearing listeners, hearingimpaired listeners and cochlear implant listeners. Audiol Neurotol 21(3):127-131.

Godar SM, Litovsky RY. (2010) Experience with bilateral cochlear implants improves sound localization acuity in children. Otol Neurotol 31(8):1287.

Gordon K, Deighton M, Abbasalipour P, Papsin B. (2014) Perception of binaural cues develops in children who are deaf through bilateral cochlear implantation. PLoS One 9(12):e114841.

Gordon K, Jiwani S, Papsin B. (2013a) Benefits and detriments of unilateral cochlear implant use on bilateral auditory development in children who are deaf. Front Psychol 4:719.

Gordon K, Wong D, Papsin C. (2013b) Bilateral input protects the cortex from unilaterally-driven reorganization in children who are deaf. Brain 136(5):1609-1625.

Grieco-Calub T, Litovsky R. (2010) Sound localization skills in children who use bilateral cochlear implants and in children with normal acoustic hearing. Ear Hear 31:645-656.

Grieco-Calub T, Litovsky R. (2012) Spatial acuity in 2-to-3-yearold children with normal acoustic hearing, unilateral cochlear implants, and bilateral cochlear implants. Ear Hear 33(5):561-572.

Grieco-Calub T, Litovsky R, Werner L. (2008) Using the observer-based psychophysical procedure to assess localization acuity in toddlers who use bilateral cochlear implants. Otol Neurotol 29(2):235-239.

Grieco-Calub T, Saffran J, Litovsky RY. (2009) Spoken word recognition in toddlers who use cochlear implants. $J$ Speech Lang Hear Res 52(6):1390-1400.

Hess C, Misurelli S, Litovsky R. (2018) Spatial release from masking in 2-year-olds with normal hearing and with bilateral cochlear implants. Trends Hear 22:233121651877556.

Kan A, Litovsky R. (2015) Binaural hearing with electrical stimulation. Hear Res 322:127-137.

Litovsky R. (1997) Developmental changes in the precedence effect: estimates of minimum audible angle. J Acoust Soc Am 102:1739-1745.

Litovsky R. (2011) Review of recent work on spatial hearing skills in children with bilateral cochlear implants. Cochlear Implants Int 12(1, Suppl):S30-S34.

Litovsky R. (2015) Development of the auditory system. In: Handbook of Clinical Neurology. Waltham, MA: Elsevier, pp. 55-72. 
Litovsky R, Godar S. (2010) Difference in precedence effect between children and adults signifies development of sound localization abilities in complex listening tasks. J Acoust Soc Am 128(4): $1979-1991$.

Litovsky RY, Gordon K. (2016) Bilateral cochlear implants in children: Effects of auditory experience and deprivation on auditory perception. Hear Res 338:76-87.

Litovsky R, Ehlers E, Hess C, Harris S. (2013) Reaching for sound measures: an ecologically valid estimate of spatial hearing in 2- to 3 -year-old children with bilateral cochlear implants. Otol Neurotol 34:429-435.

Litovsky R, Goupell MJ, Godar S, Grieco-Calub T, Jones GL, Garadat SN, Agrawal S, Kan A, Todd A, Hess C, Misurelli S. (2012) Studies on bilateral cochlear implants at the University of Wisconsin's Binaural Hearing and Speech Laboratory. J Am Acad Audiol 23(6):476-494.

Litovsky R, Johnstone PM, Godar S, Agrawal S, Parkinson A, Peters R, Lake J. (2006a) Bilateral cochlear implants in children: localization acuity measured with minimum audible angle. Ear Hear 27:43-59.

Litovsky R, Parkinson A, Arcaroli J, Sammeth C. (2006b) Simultaneous bilateral cochlear implantation in adults: a multicenter clinical study. Ear Hear 27:714-731.

Middlebrooks J, Green D. (1991) Sound localization by human listeners. Annu Rev Psychol 42:135-159.

Misurelli S. (2014) An investigation of spatial hearing in children with normal hearing and with cochlear implants and the impact of executive function (dissertation). University of Wisconsin-Madison, Madison, WI.

Misurelli S, Litovsky R. (2012) Spatial release from masking in children with normal hearing and with bilateral cochlear implants: effect of interferer asymmetry. J Acoust Soc Am 132: $380-391$.

Mok M, Galvin K, Dowell R, McKay C. (2009) Speech perception benefit for children with a cochlear implant and a hearing aid in opposite ears and children with bilateral cochlear implants. Audiol Neurotol 15:44-56.

Morrongiello B, Rocca P. (1990) Infants' localization of sounds within hemifields: estimates of minimum audible angle. Child Dev 61(4):1258-1270.
Muir D, Clifton R, Clarkson M. (1989) The development of a human auditory localization response: A u-shaped function. Can $J$ Psychol 43(2):199-216.

Murphy J, Summerfield Q, O’Donoghue G, Moore R. (2011) Spatial hearing of normally hearing and cochlear implanted children. Int $J$ Pediatr Otorhinolaryngol 75(4):489-494.

Olsho L, Koch E, Halpin C, Carter E. (1987) An observer-based psychoacoustic procedure for use with young infants. Dev Psychol 23(5):627-640.

Peng ZE, Hess C, Saffran JR, Edwards JR, Litovsky RY. (2019) Assessing fine-grained speech discrimination in young children with bilateral cochlear implants. Otol Neurotol 40(3):e191-e197.

Perris E, Clifton R. (1988) Reaching in the dark toward sound as a measure of auditory localization in infants. Infant Behav Dev 11(4):473-491.

Reeder R, Firszt J, Cadieux J, Strube M. (2017) A longitudinal study in children with sequential bilateral cochlear implants: time course for the second implanted ear and bilateral performance. $J$ Speech Lang Hear Res 60(1):276-287.

Steel M, Papsin B, Gordon K. (2015) Binaural fusion and listening effort in children who use bilateral cochlear implants: a psychoacoustic and pupillometric study. PLoS One 10(2):e0117611.

Van Deun L, Van Wieringen A, Scherf F, Deggouj N, Desloovere C, Offeciers FE, Van de Heyning PH, Dhooge IJ, Wouters J. (2009) Earlier intervention leads to better sound localization in children with bilateral cochlear implants. Audiol Neurotol 15(1): $7-17$.

Van Deun L, van Wieringen A, Van den Bogaert T, Scherf F, Offeciers F, Van de Heyning P, Desloovere C, Dhooge I, Deggouj N, Raeve L, Wouters J. (2009) Sound localization, sound lateralization, and binaural masking level differences in young children with normal hearing. Ear Hear 30(2): $178-190$.

Van Hoesel R. (2004) Exploring the benefits of bilateral cochlear implants. Audiol Neuro-Otol 9:234-246.

Zheng Y, Godar S, Litovsky R. (2015) Development of sound localization strategies in children with bilateral cochlear implants. PLoS One 10(8):e0135790. 